\title{
Tableau de la France de Jules Michelet : espace GéographiQue, HISTORIQUE OU POÉTIQUE?
}

\author{
Jaroslav STANOVSKÝ \\ Université Masaryk de Brno/Université Paris-Est
}

\begin{abstract}
En): The article deals with the notion of space in the works of historian Jules Michelet (1798-1874), more precisely in his text Tableau de la France, which is included in the Michelet's life's work: Histoire de France. The aim of the article is to present the complexity of Michelet's texts and at the same time to demonstrate the coexistence of the scientific and literary discourse which is typical for all the Michelet's works. The article presents firstly the historical thinking of Michelet in the context of the romantic historiography, most importantly his concept of "historical resurrection". Then it explains the specific position of Tableau de la France in the plan of the Histoire de France. The main part of the article comprises an analysis of three different dimensions of Tableau that correspond to three conceptions of the French space: the geographical, the poetic and the historical.
\end{abstract}

Key words (En): 19th century ; Jules Michelet ; romanticism ; space ; historiography ; narrativity

Mots-clés (Fr) : 19e siècle ; Jules Michelet ; romantisme ; espace ; historiographie ; narration

\section{Introduction}

Quelle est la notion de l'espace dans une œuvre historique ? Peut-il jouer plusieurs rôles en même temps ou fonctionne-t-il tout simplement comme une coulisse du théâtre historique ? Et la description géographique, peut-elle contenir une dimension littéraire ? Le présent article essaie de répondre à ces questions dans le cas de l'œuvre de Jules Michelet, considéré comme l'un des plus grands historiens français du $19^{\mathrm{e}}$ siècle. L'objectif de cette étude est de présenter et d'analyser un de ses textes les plus célèbres, le Tableau de la France. Nous voudrions démontrer comment le Tableau s'inscrit dans la logique de la conception historique de Jules Michelet. Ensuite, nous expliquerons la place du Tableau dans le cadre de la synthèse de l'Histoire de France. Dans la partie principale de notre article, nous insisterons sur le caractère pluridimensionnel de ce texte en présentant les différentes formes de l'espace dans le Tableau. Nous parlerons de l'espace géographique, de l'espace poétique et finalement de l'espace historique pour démontrer une certaine coexistence du discours scientifique et littéraire, ce qui est caractéristique pour l'œuvre de Michelet.

\section{Historiographie romantique, Jules Michelet et son Histoire de France}

Nous pouvons qualifier Michelet de représentant typique de l'historiographie romantique. Cette période est marquée à la fois par l'évolution progressive de la science historique et par l'accent mis sur l'histoire nationale. À cette époque, la mission de l'historien et sa position dans la société ont changé : « le renouvellement historique » a engendré de nouveaux défis. (Paul VAN TIEGHEM, 1969 : 116-118) L'historien romantique ne devait plus seulement découvrir le passé. Son objectif était aussi d'expliquer l'évolution historique de sa propre nation et même de créer 
son identité. C'est pourquoi les historiens romantiques cherchent à esquisser une représentation complexe de l'histoire d'un peuple ou d'un pays. František Palacký a traité de cette façon l'histoire tchèque, Joachim Lelewel l'histoire polonaise, Nikolaj Karamzin celle de la Russie, etc. (ČINÁTL, 2008 : 3). L'intérêt pour le passé a engendré également des conditions plus favorables pour la recherche scientifique de l'histoire. À partir de ce moment les historiens se préoccupent davantage de l'érudition, du travail critique des sources diplomatiques et narratives. Or, l'historiographie romantique se situe toujours à la frontière entre la littérature et la science, par la vision romantique du monde de même que par la création des grands concepts de l'histoire.

Le travail de Jules Michelet se distingue par ces deux traits de l'historiographie romantique, par l'importance de l'histoire nationale et par le souci d'un travail scientifique précis, associé à la recherche d'une nouvelle méthodologie. Au cœur même de son œuvre se trouve ainsi l'ouvrage de sa vie, son immense et admirable Histoire de France. Il est composé de vingt volumes, dont six consacrés au Moyen Âge (publiés de 1833 à 1844), sept à la Révolution (1847-1853) et sept aux temps modernes, c'est-à-dire du règne de Charles VIII jusqu'à celui de Louis XVI (18571867). Les volumes de la Révolution sont parfois classés en dehors du cadre de l'Histoire de France car, comme l'affirme Paule Petitier, "L'Histoire de la Révolution constitue bien une cuvre autonome, dont le sujet est la «Révolution» avant d'être la France. » (PETITIER, 2008 : 8) L'Histoire de France a été achevée par la Préface à l'édition complète de 1869. Il s'agit du texte métahistorique le plus important de Michelet. Il a été rédigé pour l'édition complète et il résume et explique nettement les opinions de son auteur sur la mission de l'historien et sur la méthodologie de l'historiographie. Michelet ne reprend pas les conceptions de ses prédécesseurs, il essaie, par contre, de formuler sa propre méthode spécifique. Cette méthode influence non seulement la recherche mais également l'écriture et tous les autres aspects de ses œuvres. C'est dans la préface de son autre ouvrage Peuple (1846) que nous trouvons le manifeste public de la conception historique selon Michelet : "Que ce soit ma part dans l'avenir, d'avoir non pas atteint, mais marqué le but de l'histoire, de l'avoir nommée d'un nom que personne n'avait dit. Thierry l'appelait narration, et M. Guizot, analyse. Je l'ai nommée résurrection et ce nom lui restera. » (MICHELET, $1946: 25)$

L'Histoire est une résurrection, voilà le credo de Michelet, repris et justifié dans sa Préface de 1869. Ce concept influence l'écriture de l'histoire sur tous ses plans. L'historien dont la mission est de faire revivre l'histoire doit s'intéresser à tous les domaines (politique, économie, géographie, culture, etc.) et donc examiner toutes les sources possibles. Il impose ainsi un tout autre point de vue sur l'Histoire : complexe et total. Le rôle clé dans le processus de l'écriture est attribué à l'imagination comme en témoigne l'image poétique de l'historien dans les archives à la fin du tome 2 de l'Histoire de France. Michelet s'adresse directement aux morts cachés dans les documents sources : "Doucement, messieurs les morts, procédons par l'ordre, s'il vous plaît. Tous vous avez le droit sur l'histoire. » (MICHELET, 1974 : 614). De même, Michelet défend le droit à la subjectivité : l'historien est celui qui réalise la résurrection et son investissement personnel dans l'œuvre est primordial. Ainsi, le narrateur est fortement impliqué dans le récit micheletiste : il 
s'exprime, il juge, il commente et de plus, il se passionne et s'extasie sans cesse. (SCHOLL, 2006 : 30) L'écriture historique de Jules Michelet se trouve ainsi à la frontière entre la science et la littérature, entre la synthèse de l'évolution historique de la France et «l'épopée lyrique », pour employer les mots d'Hippolyte Taine. Cette mise en écriture complexe de l'histoire est caractéristique également de notre texte, Le Tableau de la France, et dans la conception de l'espace français présenté par Michelet.

\section{Tableau de la France et sa place dans l'œuvre de Michelet}

Dans le contexte de l'Histoire de France, la place du texte traité est bien particulière. Les autres livres de l'Histoire de France narrent telle ou telle époque historique et ils suivent un ordre chronologique. En revanche, le Tableau de la France n'est pas ancré dans une époque concrète mais il donne la perspective totale de l'histoire française, de ses débuts jusqu'à l'époque de Michelet. Il est organisé selon un ordre spatial : la France, province par province. La description n'est pas du tout statique, mais elle excelle, au contraire, par sa dynamique. La forme choisie, le tableau (en tant que figure de style), annonce déjà le style vivant de la description et également le caractère complexe du texte. Pierre Fontanier, auteur contemporain de Michelet, définit le tableau de la façon suivante : « On appelle du nom de Tableau certaines descriptions vives et animées, de passions, d'actions, d'événements, ou de phénomènes physiques ou moraux. » (FONTANIER, 2009: 431). Michelet invite ainsi ses lecteurs à faire un tour imaginaire depuis la Bretagne en passant par l'Aquitaine et le Midi, l'est et le nord du pays pour aboutir en Île-de-France, au cœur même de la France. Il fixe ainsi son histoire dans l'espace en lui donnant un cadre géographique. Il expose également les rapports entre l'Histoire et le territoire et leurs influences réciproques.

La convention de l'écriture historique prescrit de placer la description géographique au début d'une œuvre. Tel est le cas du Tableau de la géographie de la France de Paul Vidal de La Blache qui ouvre un autre grand projet historique, l'Histoire de France depuis les origines jusqu'à la Révolution dirigé par Ernest Lavisse. Jules Michelet, en apparence, viole cette règle. Le Tableau n'est pas placé au début de l'Histoire de France mais au début du volume 2. Cependant, c'est cette partie qui doit être considérée comme le vrai début de l'œuvre, c'est cette partie qui commence véritablement l'histoire de France et le premier tome n'est que son prélude. C'est en effet aux $10^{\mathrm{e}}$ et $11^{\mathrm{e}}$ siècles qu'il faut placer, selon Michelet, la naissance de la France et l'aube de son histoire. Nous savons que Michelet se considérait comme de la Nation, conformément à l'approche romantique de l'histoire. Il ouvre ainsi le Tableau par ce passage représentatif de toute son idéologie : "L'histoire de France commence avec la langue française. La langue est le signe principal d'une nationalité. » (MICHELET, 1974 : 331)

La nation française identifiée par sa langue, voilà une répercussion caractéristique de l'esprit romantique. Par ailleurs, l'accent mis sur la langue relève de la conception de la nation: "La réalité symbolique de la langue désigne en somme la nation elle-même comme espace plus symbolique que physique.» (PETITIER, 2008 : 35) De plus, un autre facteur important participe à la formation 
du pays. Le tome 1 se termine par l'avènement d'Hugues Capet en 987. Ses successeurs, les Capétiens, sont la première dynastie nationale. Ainsi, la France a sa langue et sa propre dynastie qui est le germe de la centralisation. L'historien affirme à ce propos : "Pour la première fois elle (=la France) se produit dans sa forme géographique.» (MICHELET, 1974 : 331) La route vers l'unité nationale peut commencer, la scène pour l'intrigue est préparée, il suffit de l'exposer aux lecteurs. La place inhabituelle de la description géographique s'inscrit ainsi dans la logique de la conception de Michelet où la Nation est l'acteur fondamental de l'histoire.

\section{Texte pluridimensionnel}

Le caractère du Tableau est polyvalent ce qui est souligné déjà par son titre. Il est possible d'y distinguer au moins trois ou quatre strates et de concevoir ainsi des lectures différentes. Comment définir le Tableau de la France et quel est le rôle de l'espace français ? S'agit-il d'un traité scientifique, d'une vision romantique du paysage français ou d'une esquisse du programme général de l'Histoire de France? Difficile à dire. Ce texte représente pour Michelet un enjeu à la fois scientifique (la représentation géographique), artistique (dans le sens originel du terme « tableau») et idéologique (le rôle du Tableau pour l'ensemble de l'Histoire de France). Enfin, le Tableau excelle par son niveau stylistique et artistique qui fait de ce texte, selon Lucien Febvre, "une des plus grandes manifestations du talent de Michelet.» (FEBVRE 2014 : 317) C'est cette complexité qui fait du Tableau un vrai chefd'œuvre.

\subsection{Espace géographique}

La première lecture du Tableau révèle un traité scientifique de géographie physique et sociale, un texte à mi-chemin entre les sciences naturelles et les sciences humaines. La géographie était au début du $19^{\mathrm{e}}$ siècle un domaine sous-estimé en tant que science et son importance n'est reconnue que peu à peu. Or, Michelet comprenait le lien intime entre l'histoire et la géographie et son but premier est de présenter et d'expliquer la relation entre la terre et l'homme, l'influence du territoire sur l'Histoire. Il annonce déjà ce programme en 1826 dans son Journal des idées: « Je voudrais faire une géographie à la fois physique et politique. (...) On insisterait sur les circonstances physiologiques, physiques, botaniques (...) qui peuvent expliquer l'Histoire. » (cité selon FEBVRE, 2014 : 318)

Dès 1831, Michelet commence à explorer systématiquement et minutieusement les provinces françaises : par ses voyages, par ses lectures. Les résultats de ses observations sont présentés dans le Tableau. Nous voyons le souci d'un travail scientifique précis, l'auteur expose toute une échelle de facteurs géographiques en examinant ses rapports avec l'évolution historique. En premier lieu, il aborde la géographie physique : les montagnes ("Les montagnes du haut Limousin se lient à celles de l'Auvergne, et celles-ci avec les Cévennes. ») (MICHELET, 1974 : 345), les fleuves ( «du côté occidental, la Seine, la Loire et la Garonne descendent à l'Océan; derrière, s'écoulent la Meuse au nord, la Saône et le Rhône au midi ») (MICHELET, 1974 : 339) et les aspects du paysage en général. Michelet aborde également la géologie («la Bretagne, (...) simple quartz et granit; la verte et rude Auvergne, 
vaste incendie éteint avec ses quarante volcans» (MICHELET, 1974 : 332) et le climat en remarquant son rapport avec l'agriculture («Les zones de la France se marquent aisément par leurs produits. Au nord, les grasses et basses plaines de Belgique et de Flandre avec leurs champs de lin et de colza, et le houblon, leur vigne amère du nord.) (MICHELET, 1974 : 333) La géographie lui permet d'expliquer les conditions historiques des provinces, par exemple l'isolement de la Vendée « qui a quatorze rivières, et pas une navigable. » (MICHELET ; 1974 : 334) De nombreuses observations portent sur la géographie sociale : Michelet décrit la population française et ses variantes diverses (il parle par exemple de la frontière linguistique en Bretagne) ou l'économie (la tradition industrielle de Lyon et des Flandres). L'auteur s'intéresse à tous les aspects de la population française y compris les coutumes ou les costumes des habitants (Roland Barthes qualifie d'ailleurs Michelet de fondateur de l'ethnologie de la France - BARTHES, 1975 : 28). Par certaines observations, le texte entre même dans le champ de la sociologie. Voir par exemple la remarque de Michelet sur le rôle du christianisme dans la société bretonne : "En Bretagne, comme en Irlande, le catholicisme est cher aux hommes comme symbole de la nationalité. La religion y a surtout une influence politique. » (MICHELET, 1974 : 338-339) Aussi la géographie « culturelle »n'est-elle pas omise, l'auteur mentionne les monuments importants (les mégalithes de Carnac), les mythes régionaux (Merlin en Bretagne, Mélusine en Poitou) et la production littéraire de telle ou telle province : «La guerre du Loup et du Renard est la grande légende du nord de la France, le sujet des fabliaux et des poèmes populaires.» (MiCHELET, 1974 : 364) Il note également l'origine des auteurs pour démontrer comment leurs régions natales influencent leur écriture (par exemple Chateaubriand en Bretagne). Le Tableau présente ainsi l'image géographique de la France sous toutes ses formes possibles. Le Tableau de Michelet est encore loin du travail scientifique positiviste de Paul Vidal le Blanche et de son Tableau de la géographie de la France mais il offre un tout nouveau type du discours sur le territoire. Cette dimension du Tableau met en évidence l'apport de Michelet à la méthodologie historique. Il s'agit d'une autre forme de «l'histoire totale », à savoir du concept proposé plus tard par Jacques Le Goff dans ses réflexions méthodologiques sur l'historiographie du Moyen Âge. Selon ce concept, l'historien doit utiliser toutes les sources possibles. Le Goff se réclame de Michelet en le qualifiant de précurseur de cette approche.

\subsection{Espace poétique}

Malgré les ambitions scientifiques du texte, le Tableau contient également une dimension littéraire. La forme choisie du texte ne ressemble pas à celle d'un traité scientifique. En effet, le Tableau est présenté comme un récit de voyage, sinon comme un guide touristique. Également par cet aspect, le Tableau correspond au contexte de son époque, car ce genre était à la mode. Outre les vrais récits de voyage, il faut mentionner aussi le projet Voyages pittoresques et romantiques dans l'ancienne France dont la publication sous la direction d'Isidore Taylor commence en 1820 et qui contribue à la formation d'une vision romantique du territoire. Sur le modèle des récits de voyage, le texte est organisé comme un tour imaginaire en plusieurs étapes. L'auteur utilise plusieurs moyens pour créer l'impression d'un 
voyage réel : le Tableau est raconté à la première personne, les descriptions des diverses régions sont liées par des phrases qui évoquent le mouvement et le voyage : «Je parlerai plus tard du centre de la France ; il me tarde de pousser au midi. »; «Je pourrais entrer par le Rouergue dans la grande vallée du Midi...mais j'aime mieux entrer par Cahors. »; "Il faut que je fraye ma route vers le nord, aux sapins de Jura. » (MICHELET, 1974 : 341, 345, 346 et 360) De même, l'auteur communique directement avec ses lecteurs et il raconte parfois ses propres expériences: «Je n'oublierai jamais le jour où je partis de grand matin d'Auray, la ville sainte des chouans, pour visiter, à quelques lieues, les grands monuments druidiques de LocMaria-Ker et de Carnac. » (MICHELET, 1974 : 338) La représentation vivante et dynamique est la preuve du talent d'écrivain de Michelet, de son génie créatif. Cependant, la qualité littéraire n'est pas un objectif en soi, elle est toujours au service des intentions scientifiques. Michelet ne dresse pas non plus la liste des curiosités touristiques, son regard est celui d'un chercheur. Il imite la forme de Voyages pour une présentation plus agréable de ses idées. Cette démarche tient également à l'importance de la dimension subjective qui caractérise l'œuvre de Michelet en général.

Une autre dimension non scientifique (d'un point de vue moderne) peut être condérée comme l'expression de la sensibilité romantique. Le raisonnement de Michelet est le suivant : les conditions géographiques déterminent le caractère de telle ou telle région et celui-ci détermine la vie et le caractère de ses habitants de mille façons. Il est donc possible d'expliquer une région à travers des signes divers : par les événements et les personnages historiques, par la toponymie, l'architecture ou les mythes locaux. Comme l'historien l'affirme dans l'introduction du Tableau : «Mais il ne suffit pas de tracer la forme géographique de ces diverses contrées; c'est surtout par leurs fruits qu'elles s'expliquent, je veux dire par les hommes et les événements que doit offrir leur histoire. » (MICHELET, 1974 : 331) Michelet cherche ainsi à déchiffrer ce langage symbolique et à expliquer la relation étroite entre le pays et l'homme. Par cet effort, il crée plutôt une vision poétique et symbolique de la France. Le mot clé pour cette dimension du Tableau est «génie », utilisé pour décrire le caractère d'une région : il est employé trente fois dans le texte. Par le processus de personnification, les régions sont représentées à l'aide de qualités humaines. Lucien Febvre qualifie cette démarche de géographie psychologique et morale. (FEBVRE, 2014: 346). Présentons ici quelques exemples des régions françaises. La Bretagne, avec son climat dur, marquée par l'inhospitalité, se caractérise par le génie «d'indomptable résistance et d'opposition intrépide, opiniâtre, aveugle » (MICHELET, 1974 : 334), par l'esprit rebelle. Pour le Poitou qui se trouve à la frontière du Nord et du Midi, est caractéristique le génie mixte et contradictoire ; ses héros sont ainsi Richard Cœur-de-Lion et ses frères, ni Anglais, ni Angevins, ni Poitevins. De plus, Michelet établit souvent des liens entre le génie d'une région et sa littérature qui est, dans sa vision symbolique, «l'expression la plus élaborée du territoire. »(PETITIER, 1994 : 161) La Provence se distingue par le caractère poétique, la Bourgogne est «le pays des orateurs, celui de la pompeuse et solennelle éloquence », alors que la prose domine dans les provinces du Nord (Michelet, 1974 : 369). Par des passages similaires, Michelet fait preuve à la fois de sa curiosité de chercheur et de sa sensibilité d'artiste. L'idée que les conditions 
naturelles prédéterminent la nature des hommes et l'histoire de tel ou tel pays n'est pas originale. Nous trouvons un raisonnement analogue dans les livres de certains romanciers contemporains, tel Honoré de Balzac ou Victor Hugo. Dans « le roman breton » Béatrix, Balzac dit à propos du vieux baron du Guénic : «En lui, le granit breton s'était fait homme. (...) Son menton voulait rejoindre le nez, mais on voyait, dans le caractère de ce nez bossué au milieu, les signes de son énergie et de sa résistance bretonne. »(BALZAC, 1962 : 23) Quant à Victor Hugo, nous pouvons citer l'exemple du roman Quatrevingt treize qui offre une certaine analogie avec le Tableau par la vision symbolique de l'espace. Victor Hugo, lui aussi, explique l'opposition de la Vendée par ses conditions naturelles en démontrant la relation entre la terre (les forêts) et les hommes. L'écrivain exprime l'idée que «l'âme de la terre passe dans l'homme. » (HUGO, 2001 : 246) Par contre, cette vision romantique de la géographie n'était pas partagée par tous les chercheurs de l'époque comme le prouve l'exemple de l'historien tchèque František Palacký. Ce contemporain de Michelet, considéré comme «le créateur de l'histoire tchèque », a exprimé un avis critique par rapport au «génie » de tel ou tel pays sans toutefois nier un certain déterminisme géographique :

Même si nous ne voulons pas du tout affirmer que les peuples tirent leur nature physique et morale des conditions, de la position et du climat des pays qu'ils habitent, nous ne pouvons pas nier que les possibilités, les avantages et les désavantages donnés par la nature de chaque pays, influencent puissamment la vie nationale et son développement, au contraire cela nous semble évident. (PALACKÝ, 1939: 14).

Si Palacký reste toujours dans le cadre de la recherche scientifique, Michelet montre plutôt par ce concept la sensibilité d'un artiste, d'un poète.

Le dernier trait de la sensibilité romantique est l'imagination de Michelet et ses manifestations diverses. Il s'agit d'ailleurs d'un trait caractéristique du style d'écriture de Jules Michelet en général. Rappelons le compte-rendu de L'Histoire de France VII d'Hippolyte Taine qui décrit l'ouvrage, justement pour sa force poétique, comme «l'épopée lyrique de la France » (cité selon CARRÉ, 1926 : 121). Celle-ci serait construite par des images et des associations qui changent complètement le ton du discours. Il semble que chez Michelet «le regard analytique génère la contemplation esthétique » (PETITIER 1994 : 249). Le texte du Tableau se caractérise ainsi par l'insertion de passages lyriques, remplis d'images poétiques. Le style de Michelet se distingue par sa richesse, par le besoin de varier le langage et par le refus de la simplicité, traits qui l'éloignent du discours scientifique proprement dit. Citons au moins deux extraits représentatifs pour cette dimension du texte. Quand l'auteur parvient dans son voyage imaginaire à la limite de la Bretagne, il s'arrête pour évoquer l'ambiance d'une façon impressionnante. Michelet emploie toutes sortes des figures stylistiques et poétiques : l'hyperbole, la répétition, le contraste (la terre et la mer) et même les allusions et métaphores bibliques (mots de Jésus Christ à Gethsémani, image de Caïn qui représente la dureté des Bas-Bretons) : 
Rien de sinistre et formidable comme cette côte de Brest ; c'est la limite extrême, la pointe, la proue de l'Ancien Monde. Là, les deux ennemis sont en face : la terre et la mer, l'homme et la nature. (...) Et même dans les moments de trêve, quand l'Océan se tait, qui a parcouru cette côte funèbre sans dire ou sentir en soi : Tristis usque ad mortem ? (...) L'homme est dur sur cette côte. Fils maudit de la création, vrai Caïn, pourquoi pardonnerait-il à Abel ? (MicHELET, 1974 : 336)

Dans une autre partie du texte, l'auteur parle de la transhumance des bergers comme d'un élément important qui caractérise la région du Midi et des Pyrénées. Le passage cité documente également la méthode de travail de Michelet : avant la publication du Tableau, il n'avait jamais visité les régions du Midi et il devait recourir aux sources disponibles (les statistiques des départements, les récits des voyageurs). En se basant sur leur étude minutieuse, l'historien a mis en œuvre son imagination et son talent stylistique pour créer un texte d'une beauté extraordinaire. :

La vie voyageuse des bergers est un des caractères pittoresques du Midi. Vous les rencontrez montant des plaines du Languedoc aux Cévennes, aux Pyrénées, et de la Crau provençale aux montagnes de Gap et de Barcelonnette. Ces nomades, portant tout avec eux, compagnons des étoiles, dans leur éternelle solitude, demi-astronomes et demi-sorciers, continuent la vie asiatique, la vie de Loth et d'Abraham, au milieu de notre Occident. (MicHELET, 1974 : 348)

Au niveau factuel, l'observation de Michelet sur l'importance de ce phénomène est tout à fait juste, attestée même par les historiens modernes (par exemple par Emmanuel Le Roy Ladurie dans sa monographie Montaillou, un village occitan). Ce qui est singulier néanmoins dans le texte du Tableau, c'est l'image poétique construite avec des éléments typiques du discours micheletiste : rythme rhétorique, reprises, métaphores, analogies. Nous pourrions en donner d'autres exemples. Néanmoins, les deux extraits précités attestent une grande qualité esthétique qui est propre au Tableau de la France et qui se différencie nettement du discours scientifique proprement dit. Cette qualité esthétique forme, avec certains éléments de la sensibilité romantique (surtout avec le concept du « génie » particularisant les différentes provinces) la dimension de «l'espace poétique» du Tableau de la France. Par elle, le Tableau se distingue nettement des textes géographiques modernes. Pourtant, ce fait ne suffit pas à attribuer une nature littéraire au texte analysé. La fonction esthétique, fortement impliquée dans le texte, n'est que secondaire et c'est la fonction informative qui prédomine.

\subsection{Espace historique}

La dernière dimension du Tableau consiste en son rôle globalisant, en l'image qu'il donne de la France et de son histoire en général. L'espace français y figure comme une scène du processus historique de l'évolution nécessaire vers l'unité et la liberté. Le Tableau est remarquable par la cohérence de son plan qui annonce déjà le plan général de l'Histoire de France. Michelet cherche à décrire la France sur deux axes qui ne sont pas séparables, géographique et historique. "La géographie est une histoire fixe et permanente et l'histoire une géographie fugitive et transitoire. » (PETITIER, 1994 : 153) Cette perspective globale est motivée par l'idée fondamentale du Tableau : saisir à la fois les relations entre le temps et l'espace et 
la proportion entre les parties et la totalité, entre les provinces et la France entière. Michelet expose la diversité étonnante de la France pour prouver sa cohérence interne. D'où la vision métaphorique à la fin du Tableau : un pays qui fonctionne comme un organisme vivant, comme le corps humain :

La force et la beauté de l'ensemble consistent dans la réciprocité des secours, dans la solidarité des parties, dans la distribution des fonctions, dans la division du travail social. La force résistante et guerrière, la vertu d'action est aux extrémités, l'intelligence au centre. (MichELET, 1974 : 381)

La France est ainsi une personne et les provinces en sont les membres. En poursuivant cette métaphore biologique, Michelet affirme : «Les nations peuvent se classer comme les animaux» (MICHELET, $1974: 383$ ) et l'espèce la plus haute, la plus évoluée est pour lui une nation-personne. Il justifie ainsi la position privilégiée de la France par rapport aux autres pays. En effet, le Tableau est aussi bien la description géographique de la France que l'image de son devenir. Ce concept cache également le principe philosophique de l'Histoire : l'humanité (dans ce cas-là limitée à la nation française) qui se créé peu à peu sur le chemin du progrès (et de l'unité). Ce n'est pas par hasard que les grandes idées du Tableau de la France sont reprises par la Préface de 1869. Il s'agit par exemple du déterminisme géographique («Tel le nid, tel l'oiseau. Telle la patrie, tel l'homme») et de l'anthropomorphisme du territoire français aux yeux de l'historien («Le premier, je la vis comme une âme et une personne. ») (MICHELET, $1974: 23$ ) Le plan du Tableau annonce ainsi le plan de toute l'Histoire de France. Michelet y dresse les grandes lignes de son histoire, les points principaux de son intrigue. La filiation de l'espace et du temps dans la conception de Michelet et la configuration de la structure du Tableau en guise de plan global du projet est la preuve de son génie créateur. Cette composition réfléchie relève de la conception historique de Michelet et de ses idées philosophiques. Néanmoins, nous pouvons la considérer également comme «la création » de l'histoire de France. Michelet a pris la matière première (le territoire de la France), il l'a organisée et l'a inscrite dans une perspective temporelle, ce qui est le signe de son talent d'écrivain et une marque de la littérarité de l'Histoire de France. Roland Barthes propose la métaphore de l'expérience chimique : le recensement des matériaux, des substances diverses (les provinces) pour former la substance définitive (le génie de la France) pendant un long processus chimique (l'histoire française). (BARTHES, $1995: 30$ et 31)

\section{Conclusion}

Dans son étude sur la géographie dans l'œuvre de Michelet, Paule Petitier désigne le Tableau comme un hapax en tant que discours sur le territoire. (PETITIER, 1994 : 9) Nous sommes d'accord avec ce jugement, il s'agit d'un récit unique, sans précédent. Il prouve à la fois la curiosité scientifique de Michelet pour différents domaines et son talent stylistique qui se manifeste dans tout son éclat. L'érudition de l'auteur s'associe à sa verve poétique. La notion de l'espace français dans le texte du Tableau est ainsi d'une grande complexité et elle contient plusieurs dimensions qui ne sont pas séparables les unes des autres. Pour reprendre la question principale de notre article, nous pouvons dire que « la France » est représentée dans le Tableau 
de Michelet par trois types d'espace qui s'entrecroisent. Sous la plume de l'historien, la France se présente d'abord comme un espace géographique: la recherche de Michelet est bien fondée (pour son époque) et la fonction du texte est tout d'abord, il faut insister sur ce point, informative. En même temps, l'espace de la France est « poétisé », d'abord par la vision proprement romantique du territoire, à savoir par le concept du «génie » des différents domaines, ensuite par le style riche de l'auteur, saturé par son imagination vivante. Rappelons également que toute la conception de la France est, en effet, fondée sur l'image du corps humain ce qui est d'ailleurs en accord avec le point de vue métaphorique qui caractérise toute l'œuvre de Michelet. Et finalement, la France dans le Tableau est également « historique ». Le Tableau joue un rôle symbolique primordial dans l'ensemble de l'Histoire de France. Par ce texte, Michelet prépare les coulisses où se déroulera le drame historique de l'évolution de la France vers l'unité et la liberté et il dresse, pour citer encore une fois Lucien Febvre, «la porte triomphale qui ouvre les siècles. »(FEBVRE, 2014 : 311) La France de Michelet est ainsi un espace complexe, pluridimensionnel. C'est cette complexité que nous avons essayé de démontrer dans notre article et qui fait du Tableau un exemple de l'évolution de la science au $19^{\mathrm{e}}$ siècle de même qu'un vrai chef-d'œuvre de la littérature romantique.

\section{BIBLIOGRAPHIE}

BARTHES, Roland (1995), Michelet, Paris, éditions du Seuil.

CARré, Jean-Marie (1926), Michelet et son temps, Paris, éditions Librairie académique.

ČInÁTL, Kamil (2008). Dějiny a vyprávění, Palackého dějiny jako zdroj historické obraznosti národa (Histoire et Narration, Histoire de Palacky comme une source de l'imaginaire historique du peuple), Prague, Université Charles (thèse de doctorat d'université).

DE BALZAC, Honoré (1962), Béatrix, Paris, éditions classiques Garnier.

FEBVRE, Lucien (2014), Michelet, Créateur de l'histoire de France, Cours au Collège de France, 1943-1944, Paris, éditions La Librairie Vuibert.

FONTAINIER, Paul (2009), Les figures du discours. Paris, éditions Flammarion.

HugO, Victor (2001), Quatrevingt treize., Paris, éditions Gallimard.

LE GoFf, Jacques. (1974), Michelet et le Moyen Âge, aujourd'hui, in : VIALLANEIX Paul (éd.), Michelet, Jules, Euvres complètes IV, Paris, éditions Flammarion, 1974, pp. 45-63.

MicheleT, Jules (1946), Le Peuple, Paris, éditions M. Didier.

Michelet, Jules (1974), Histoire de France, Tome 2. in : ViallaneiX Paul (éd.), Michelet Jules., CEuvres complètes IV, Paris, éditions Flammarion, 1974, pp. 696-727.

PALACKÝ, František (1939), Dějiny národa českého v Čechách a na Moravě (L'Histoire du peuple tchèque en Bohême et Moravie), volume 1, Prague, éditions L. Mazáč.

Petitier, Paule (1994), La Géographie de Michelet. Territoire et modèles naturels dans les premières auvres de Michelet, Paris/Montréal, éditions l'Harmattan. 
PetiTIER, Paule (2008), Préface à l'édition de l'Histoire de France, in : MiCHELET Jules, Histoire de France II. Tableau de la France, Les Croisades, Saint Louis, Sainte-Marguerite-sur-Mer, éditions Equateurs.

Résurrection de Michelet (table ronde), in : Romantisme, 10, Michelet cent ans après, pp. 13-46.

SCHOLL, Dorothea (2006), Visions de la Renaissance et du Baroque chez Michelet, in : ClAudon, Francis; ENCREvé, André; RichiER, Laurent (dir.), L'Historiographie romantique, Paris, éditions Bière.

VAn TIEGHEM, Paul (1969), Le romantisme dans la littérature européenne, Paris, éditions Albin Michel. 